\title{
Variations In Ada Levels In Various Body Fluids- An Original Study
}

\author{
Dr.Shivanand Gundalli ${ }^{1}$, Dr. Rutuja kolekar ${ }^{2}$. \\ Assistant Professor Department Of Pathology MRMC Hyderabad ${ }^{l}$, Assistant ProfessorDepartment Of \\ Obstetrics And Gynaecology LTMC Sion Mumbai ${ }^{2}$
}

\begin{abstract}
:
Aim: The aim of our study was to determine the frequency of various types of basal cell carcinomas encountered in our practice, to delineate the spectrum in our setup and to determine the different histological patterns, anatomical location, site predilection, and age and sex incidence.
\end{abstract}

\section{Introduction}

Adenosine deaminase levels : enzyme of purine metabolism that deaminates adenosine and deoxy adenosine to ionosine and 2 deoxy ionosine respectively.Ada defeciency leads to SCID .the abnormality of this enzyme is associated with hereditary non spherocytic hemolytic anaemia .it is only rbc enzymopathy that is inherited as autosomal dominant disorder.(tietz biochemistry)More ada activity is present in cytoplasm of $t$ cells than cytoplasm of $\boldsymbol{B}$ cells. Ada activity is usually elevated in early hiv infection as a result of stimulation.

(Wiliams Haematology)

Cut Off Value

Csf -10

Serum -18

Pleural Fluid- 33u/L

Ada Sample Is Stable For 7 Days At 2-8degree Centigrade.

Interefernces: Hemolysis Interefernces With Ada Level Lipaemia And Biliru Bin Don T Interfere.

Ada can be elevetaed in liver patients referrance;kobayashi F ikoda T, Marumo F ,Sato C .Adenosine deaminase isoenzymes in liver disease.Am J Gastroenterol 1993;88:226-71.

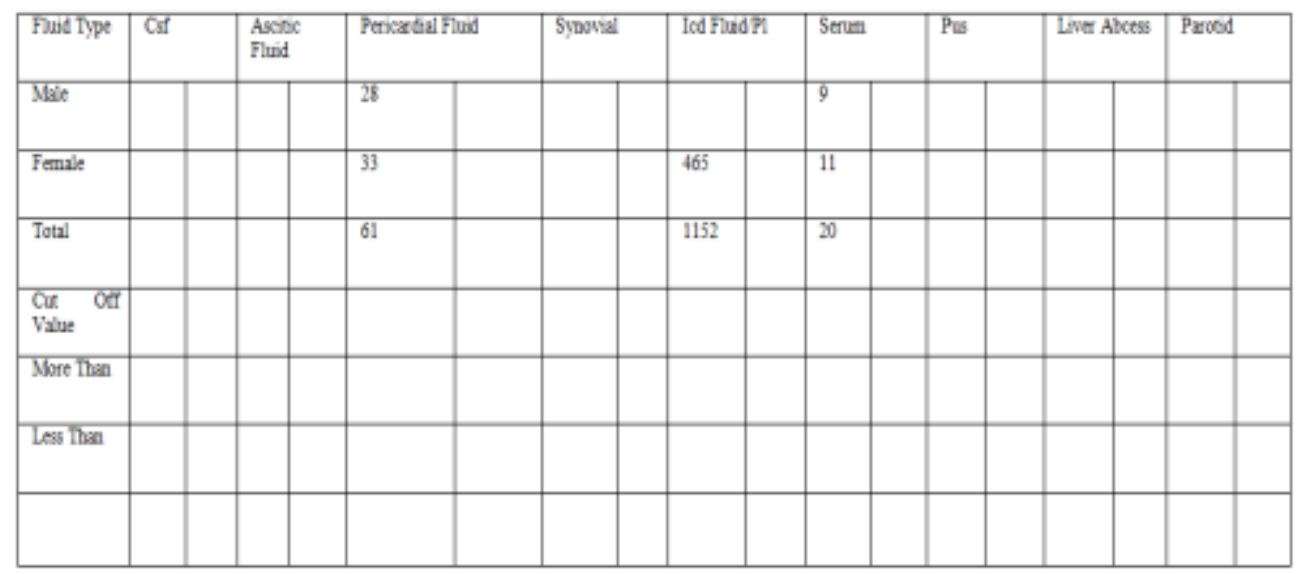

Total ada 5418 samples

Male -3839

Female-1589

ICD FLUID.-6 ADA-CSF-1450 Ascitic Fluid-700 Peritoneal Fluid-14 Synovial -10 Serum-20 Pus-8 All R More Than 200

Liver Abcess- 2

Parotid -1 Capd Fluid-3

More than 36-3839 csf More than 36-50

Liver abcess

More Than 500-2 Capd Fluid Less Than 2

Ada

Total--3839 Male-2245 .More Than 36 Is 1152.

Pericardial- 61 Male -28 Fema-33 
Less Tahn 1.00 Value-36

Less Tahn Value-36-----1081 Normal

More Tahn Value-36----1152

Csf Ada Noraml Is 0-6

In Which One Is More Than 6

Peritonea Fluid Total -11 Out Of Which 4 Have More Than 36 Rest Normal.

Synovial Fluid Is 9 Out Of This 6 Is More Than 36.

Serum Ada -Total Is 20

9 Male 11 Female

Normal Reporting Is More Than 60 Detected .40-60 Indeterminate ..

More Than 60 Is 3 Only

Omkar Is 23 Samples .

Total Plueral Fluid Is 1152 . Out Of This 461 Female

Ascitic Is 698

Cyst Aspiration Fluid\# (Fluid Vol - $40 \mathrm{Ml}$ ) -1 Value Is 35

Age Less Than 10yrs Is 40 . Age 0 Is 43.

14 Years Is 35

Capd Fluid Range Not Defined Toatl -3 Value 4,8,Less Than 1.

Adenosine deaminase levels : enzyme of purine metabolism that deaminates adenosine and deoxy adenosine to ionosine and 2 deoxy ionosine respectively.Ada deficiency leads to SCID .the abnormality of this enzyme is associated with hereditary non spherocytic hemolytic anaemia .it is only rbc enzymopathy that is inherited as autosomal dominant disorder.(tietz biochemistry) More ada activity is present in cytoplasm of $t$ cells than cytoplasm of $\boldsymbol{B}$ cells. Ada activity is usually elevated in early hiv infection as a result of stimulation.

(Wiliams Haematology)

Cut Off Value

Csf -10

Serum -18

Pleural Fluid- 33u/L

Ada Sample Is Stable For 7 Days At 2-8degree Centigrade.

Interefernces: Hemolysis Interefernces With Ada Level Lipaemia And Biliru Bin Don T Interfere.

Ada can be elevetaed in liver patients referrance;kobayashi $\mathrm{F}$ ikoda $\mathrm{T}$, Marumo $\mathrm{F}$,Sato $\mathrm{C}$.Adenosine deaminase isoenzymes in liver disease.Am J Gastroenterol 1993;88:226-71.

Exclusion Criteria: Patients undergoing treatment for any thyroid disorders, patients taking lipidlowering drugs, patients with diabetes, patients with malignancy, and pregnant women were excluded.

Collection and Analysis of Blood Samples: Informed consent was obtained from all subjects for participating in the study. Blood samples were collected by venipuncture using an aseptic technique. The serum separated from the samples was analyzed for following biochemical parameters.

Blood samples were analyzed for: Blood glucose fasting by using enzymatic glucose oxidasehorseradish peroxidase-based end-point method. HbA1c was determined by ion-exchange resin method.

Serum separated from the samples was analyzed for: Thyroid function tests (T3, T4, and TSH) by RIA method. Fructosamine test was conducted using nitroblue tetrazolium method.

\section{Materials And Methods}

Activity of ADA was determined in 60 children of age range 6 months to 11 years admitted to department of pediatrics at the Postgraduate Institute of Medical Education and Research, Chandigarh, India during 1997-98. Clinical and laboratory evidence of meningitis/ meningoencephalitis was taken as inclusion criteria. Enzyme activity was measured in CSF of four groups of patients i.e., TBM, PTM, AM and PM. These four groups were characterised as given below. Pyogenic meningitis (PM) groupIn this group, CSF of patients showing organisms in gram stained smear or culture or presence of bacterial antigen on latex agglutination was takenIndian Journal of Clinical Biochemistry, 2004, 19 (2) 5-9Indian Journal of Clinical Biochemistry, 20046 as diagnostic criteria. In the absence of organisms CSF showed pleocytosis of more than 100 cells/ mm3 predominantly polymorphs, sugar less than half of blood sugar and protein more than $60 \mathrm{mg} \%$. This was taken as inclusion criteria. Partially treated pyomeningitis (PTM) group This group consisted of patients in whom their CSF showed presence of organisms on gram stained smear or culture or presence of bacterial antigen. In the absence of organism CSF showed pleocytosis of more than 100 cells $/ \mathrm{mm} 3$, sugar less than half of blood sugar, protein more than $60 \mathrm{mg} \%$ and who had received I.V. antibiotics for pyogenic meningitis for more than 48 hours before coming to hospital. Aseptic meningitis (AM) group This group consisted of patients whose CSF showed absence of organisms on gram stain or culture and CSF pleocytosis with more than 10 cells $/ \mathrm{mm} 3$, 
predominantly lymphocytes and sugar more than $2 / 3$ of blood sugar value. Tuberculous meningitis (TBM) group In this group the patients had two or more of the

following features on history; fever for > two weeks, contact with an adult with tuberculosis, positive Mantoux test. This group had CSF with absolute lymphocyte counts $>50$ cells $/ \mathrm{mm} 3$, protein more than $60 \mathrm{mg}$ $\%$ and sugar less than $2 / 3$ of blood sugar, chest X-ray showing skiagram suggestive of pulmonary TB, isolation of AFB from any site, CT scan showing evidence of chronic meningitis like hydrocephalus, basal exudates, infarcts, tuberculomas and histological evidence of tuberculosis. Activity of ADA was assayed according to the method of Guisti (9). Two ml of CSF sample was collected in a sterile bottle at the time of admission. Patients in whom the lumbar puncture was traumatic were excluded from the study. The samples obtained were centrifuged at $2000 \mathrm{~g}$ form $10 \mathrm{~min}$ and the supernatant stored at $-20^{\circ} \mathrm{C}$ until estimation. One unit of activity represented the deamination of one micromole of adenosine $/ \mathrm{min}$ at $37^{\circ} \mathrm{C}$ and was expressed as U/L. Statistical Analysis The means \pm standard error (SE) of ADA values was calculated for each group of subjects. Wilcoxon's rank sum test was used to determine

statistical significance. Pearson's formula was used to calculate the coefficient of correlation (r-value).

\section{Reagent}

Microxpress Ada-Mtb Is A Reagent For Laboratory Use Only.

Ada-Mtb Comprises Of:

a) Ada-Mtb Reagent (L1) - Buffer Reagent, Ready To Use

B) Ada-Mtb Reagent (L2) - Adenosine Reagent, Ready To Use

C) Ada-Mtb Reagent (L3) - Phenol Reagent

D) Ada-Mtb Reagent (L4) - Hypochlorite Reagent

E) Ada-Mtb Standard (S) - Ada Standard, Ready To Use

\section{Principle}

Adenosine Deaminase hydrolyses adenosine to ammonia and inosine. The ammonia formed further reacts with a phenol and hypochlorite in an alkaline medium to form a blue indophenol complex with sodium nitroprusside acting as a catalyst. Intensity of the blue coloured indophenol complex formed is directly proportional to the amount of ADA present in the sample.



It is recommended that each laboratory establish its own normal range representing its patient population.

\section{Storage And Stability}

(1) Store the ADA-MTB kit at $2-8^{0} \mathrm{C}$, away from light. (2) Stability of the ADA-MTB kit is as per the expiry date mentioned on the label.

\section{Note}

(1) It is important that kit components from the same lot are used for achieving accurate and reproducible results. Do not intermix reagents from different lots. (2)The sequence of addition of reagents should be followed meticulously for achieving accurate results.

\section{Additional Material Required}

Test tubes, test tube stand, waterbath/incubator $\left(37^{\circ} \mathrm{C}\right)$, distilled or deionised water, variable volume pipettes, spectrophotometer with filter at $570-630 \mathrm{~nm}(\mathrm{Hg} 578$ or $623 \mathrm{~nm})$ at $37^{\circ} \mathrm{C}$ or colorimeter with yellow or red filter, stopwatch. 


\section{Reagent Preparation}

Reagents L1, L2 and standard are ready to use. Adenosine Reagent ( L2 ) may form crystals at $2-8^{0}$ C. Dissolve the same by gently warming the reagent for some time before use. Both the Phenol Reagent (L3) \& Hypochlorite Reagent (L4) need to be diluted 1: 5 with distilled water before use (1 part of reagent +4 parts of distilled water). The Working Phenol Reagent and Working Hypochlorite Reagent are stable for at least 6 months when stored at $2-8^{0} \mathrm{C}$ in tightly closed bottles.

\section{Specimen Collection And Preparation}

Collect specimen prior to use of antimicrobial agent. Wherever possible, indicate clearly that patient is on antitubercular drugs.

CSF: Collect as much as possible in a syringe, clean skin with alcohol before aspirating specimen.

Body fluids: Disinfect the site and collect specimen with aseptic precautions.

Serum, Plasma: No special preparation of the patient is required prior to sample collection by approved techniques. It is recommended to use fresh sample specimen for testing. Do not use haemolyzed, contaminated or turbid sample specimens. Fresh EDTA, citrate, heparinised or oxalate anticoagulated plasma specimens are suitable for performing the test.

Ada is reported to be stable in serum for 3 days at $2-8^{0} \mathrm{C}$ and in biological fluids for 2 days at $2-8^{0} \mathrm{C}$, as after this, ammonia may be released in the samples even without any microbial contamination.

\section{Test Procedure}

1. Bring all reagents and samples to room temperature before use.

2. Prepare the Working Phenol Reagent and Working Hypochlorite Reagent.

3. Set the spectrophotometer filter at $570-630 \mathrm{~nm}(\mathrm{Hg} 578$ or $623 \mathrm{~nm})$ at $37^{0} \mathrm{C}$.

4.Pipette into clean dry test tubes labeled Blank (B), Standard (S), Sample Blank (SB) and Test (T) as follows:

\begin{tabular}{|l|l|l|l|l|}
\hline Addition Sequence & $\begin{array}{l}\mathrm{B} \\
(\mathrm{ml})\end{array}$ & $\begin{array}{l}\mathrm{S} \\
(\mathrm{ml})\end{array}$ & $\begin{array}{l}\mathrm{SB} \\
(\mathrm{ml})\end{array}$ & $\begin{array}{l}\mathrm{T} \\
(\mathrm{ml})\end{array}$ \\
\hline Buffer Reagent & 0.20 & 0.20 & - & - \\
\hline Adenosine Reagent & - & - & 0.20 & 0.20 \\
\hline Deionised water & 0.02 & - & - & - \\
\hline Standard & - & 0.02 & - & - \\
\hline Sample & - & - & - & 0.02 \\
\hline
\end{tabular}

5. Mix well and incubate at $37^{\circ} \mathrm{C}$ for exactly 60 minutes, and then add the following:

\begin{tabular}{|l|l|l|l|l|}
\hline Working Phenol Reagent & 1.00 & 1.00 & 1.00 & 1.00 \\
\hline Sample & - & - & 0.02 & - \\
\hline Working Hypochlorite Reagent & 1.00 & 1.00 & 1.00 & 1.00 \\
\hline
\end{tabular}

6. Mix well and incubate at $37^{\circ} \mathrm{C}$ for 15 minutes or at R.T. for 30 minutes.

7. Measure the absorbance of the Blank (Abs. B), Standard (Abs. S), Sample Blank (Abs. SB) and Test (Abs. T) against distilled water.

\section{Calculations}

Abs. T - Abs. SB

Total ADA activity in $\mathrm{U} / \mathrm{L}=$ X 50

Abs. S - Abs. B

\section{Linearity}

The procedure is linear upto $150 \mathrm{U} / \mathrm{L}$. If values exceed this limit dilute the sample with deionised water and repeat the assay.Calculate the value using the appropriate dilution factor.

\section{Remarks}

1. One unit of ADA activity releases three nanomoles of ammonia in the reaction in 1 hour at $37^{0} \mathrm{C}$.

2. Patients with hyperamoniemia, kidney disorders and hepatitis can present high levels of ADA values. Patients with chronic malnutrition or HIV can present low levels of ADA values.

3. Using a cut off level of 60 units/L of ADA, values has been reported to show the Specificity and the Sensitivity of the test as above $90 \%$ for the MTB infection.

4. Below 60U/L of ADA, the serum ADA specificity and sensitivity is lower and should be interpreted in the light of other tests for confirmation of Mycobacterium tuberculosis infection. 


\section{Summary}

Tuberculosis occurs worldwide and is rampant in many countries. Though curable, its infection is on the rise. The most specific test is the positive bacterial culture of a patient's sputum sample. This is cumbersome and time consuming. X-rays, smears for AFB and Tuberculin tests though comparatively rapid are not conclusive. Adenosine Deaminase (ADA) is an enzyme widely distributed in mammalian tissues, particularly in T Lymphocytes. Increased levels of ADA are found in various forms of tuberculosis making it a marker for the same. Though ADA is also increased in various infectious diseases like Infectious Mononucleosis, Typhoid, Viral Hepatitis, initial stages of HIV, and in cases of malignant tumours, the same can be ruled out clinically.

[1]. Jose, L., Banales, M.D., et. al. (1991), Chest 99/2: 355

[2]. Jose, M. Martinez-Vazquez, et. al. (1986), Gut $27: 1049$ - 1053.

[3]. Imma Ocana, et. al. (1986), Thorax $41: 888$ - 889.

[4]. Giusti, G., Galanti, B., Methods of Enzymatic Analysis, Pg. 1092 - 1099.

[5]. Lutwick, L.I., Tuberculosis, Chapman Hall Medical, 1st. Ed. Pg. 175.

[6]. Diagnostic Value of ADA and its Isoenzyme in Tuberculosis effusions, Dept. of Internal Medicine, Shiraz E- Medical Journal.

[7]. Data on file: Tulip Diagnostics. 